\section{FRI0522 MITOCHONDRIAL BACKGROUND IMPACT ON THE JOINT DEGENERATION PROCESS DURING AGING AND FORCED EXERCISE: A CONPLASTIC MOUSE MODEL}

Morena Scotece ${ }^{1}$, Ignacio Rego-Perez ${ }^{1}$, Ana Victoria Lechuga Vieco ${ }^{2}$ Purificación Filgueira-Fernández ${ }^{1}$, Jose Antonio Enriquez ${ }^{2}$, Francisco J. Blanco ${ }^{1}$. ${ }^{1}$ Servicio de Reumatología. Instituto de Investigación Biomédica de A Coruña (INIBIC). Complexo Hospitalario Universitario de A Coruña (CHUAC), Sergas. Universidade da Coruña (UDC), A Coruña, Spain; ${ }^{2}$ Grupo de Genética funcional del sistema de fosforilación oxidativa. Centro Nacional de Investigaciones Cardiovasculares (CNIC), Madrid, España, Madrid, Spain

Background: Several studies indicated that osteoarthritis has a strong genetic component with a prevalent role of mitochondria and mtDNA variations (1)

Objectives: In the present study, we aimed to investigated the influence of the mtDNA variation in the joint deterioration using mice with the same nuclear genome but different mtDNA variants (named conplastic mice) (2) during aging and forced exercise.

Methods: Conplastic mice (BL/6 ${ }^{\mathrm{NZB}}$ ) strain was developed with the C57BL/6JOlaHsd nuclear genome and the NZB/OlaHsd mtDNA to compare with the original C57BL/6JOlaHsd strain $\left(\mathrm{BL} / 6^{\mathrm{C} 57}\right)$. Knee joints from $\mathrm{BL} / 6^{\mathrm{NZB}}$ mice as well as from $\mathrm{BL} / 6^{\mathrm{C} 57}$ mice were processed and cut into coronal sections. The mice were sacrificed at 25,75 and 90 weeks of age and knee joints were collected for histological analysis. All sections were stained with Hematoxylin-Eosine and Safranin O-fast green and graded using a Mankin scoring system. Another group of mice from both $\mathrm{BL} / 6^{\mathrm{NZB}}$ and $\mathrm{BL} / 6^{\mathrm{C} 57}$ strains were subjected to exercise by running in a treadmill $400 \mathrm{~m} /$ day three times a week. After 75 and 90 weeks of age, mice were sacrificed and knee joints were processed for histological analysis. Cartilage expression of markers of autophagy like LC3 and metalloproteinases like MMP-13 were also analysed by immunohistochemistry in both strains. The results are given as mean \pm SEM and statistical analysis was performed using non parametric unpaired t-test (Graph Pad Prism $\checkmark$ 6.0).

Results: In response to aging, conplastic mice $\mathrm{BL} / 6^{\mathrm{NZB}}$ presented reduced cartilage Mankin score at $25(\mathrm{p}=0.0079), 75(\mathrm{p}=0.0087)$ and $90 \quad(\mathrm{p}=0.064)$ weeks when compared with mice of the original strain $\mathrm{BL} / 6^{\mathrm{C57}}$ at the same age. Specifically, we showed a reduced score in both femoral condyle (FC) and tibial plateau (TP) of BL/6 $6^{\mathrm{NZB}}$ mice that reached the statistical significance at 25 (FC: $\mathrm{p}=0.0317$; TP: $\mathrm{p}=0.0079$ ), 75 ( $F \mathrm{C}: \mathrm{p}=0.0411$; TP: $p=0.0238$ ) and borderline the statistical significance at 90 (FC: $\mathrm{p}=0.0649$; TP: $\mathrm{p}=0.0628$ ) weeks of age. These results were accompanied with more expression of LC3 in cartilage from BL/6 ${ }^{\mathrm{NZB}}$ mice at 75 weeks when compared with cartilage from $\mathrm{BL} / 6^{\mathrm{C} 57}$ at the same age $(\mathrm{p}=0.0152)$. We also reported a significant decrease of LC3 expression in cartilage from mice at 75 weeks when compared with mice at 25 weeks in both strains confirming the decrease of autophagy with aging. Difference in MMP13 cartilage expression between the two mice strains were also found. In the mice subjected to exercise, $\mathrm{BL} / 6^{\mathrm{C} 57}$ presented an increased cartilage score in the medial compartment $(p=0.0286)$ and lateral compartment $(p=0.057)$ of the joint at 90 weeks when compared with $B L / 6^{\text {NZB }}$ mice at the same age.

Conclusion: This study demonstrated that aging and forced exercise in conplastic mice BL/6 $6^{\mathrm{NZB}}$ are associated with a reduced joint deterioration compared with the original strain $\mathrm{BL} / 6^{\mathrm{C} 57}$. Moreover, we showed that mtDNA variants can improve the aging process at joint level through the modulation of autophagy. These results support the hypothesis that mtDNA background has a role in the process of joint damage, suggesting that mtDNA has potential as novel therapeutic target in OA associated to aging.

\section{REFERENCES:}

[1] Mitochondrial DNA variation and the pathogenesis of osteoarthritis phenotypes. Nat Rev Rheumatol. 2018 Jun;14(6):327-340

[2] Mitochondrial and nuclear DNA matching shapes metabolism and healthy ageing; Latorre-Pellicer, et al. Nature2016;535(7613):561-5

Disclosure of Interests: Morena Scotece: None declared, Ignacio RegoPerez: None declared, Ana Victoria Lechuga Vieco: None declared, Purificación Filgueira-Fernández : None declared, Jose Antonio Enriquez: None declared, Francisco J. Blanco Consultant for: AbbVie, Bioiberica, BMS, GSK, Grünenthal, Janssen, Lilly, Pfizer, Regeneron, Roche, Sanofi, TRB Chemedica, and UCB

DOI: 10.1136/annrheumdis-2019-eular.3201

\section{FRI0523 \\ TLR-1/2 SIGNALING IMPAIRS MITOCHONDRIAL OXIDATIVE PHOSPHORYLATION IN CHONDROCYTES VIA THE INDUCTION OF NITRIC OXIDE}

Ping Shen ${ }^{1}$, Michael Fuchs ${ }^{2}$, Marie Reisener ${ }^{3}$, Clemens Gwinner ${ }^{3}$, Peihua Wu', Tobias Jung ${ }^{3}$, Matthias Pumberger ${ }^{3}$, Carsten Perka ${ }^{3}$, Max Löhning ${ }^{1} .{ }^{1}$ Deutsches Rheuma-Forschungszentrum, Pitzer Laboratory of Osteoarthritis Research, Berlin, Germany, ${ }^{2} R K U$ university hospital Ulm, Orthopedic surgery, Ulm, Germany, ${ }^{3}$ Charité - Universitätsmedizin Berlin, Centrum für Muskuloskeletale Chirurgie (CMSC), Berlin, Germany

Background: Osteoarthritis $(O A)$ is a degenerative disease that causes progressive loss of joint function, representing a severe health problem plaguing this ageing world. Cartilage matrix degradation and catabolic factor production are closely associated with $O A$ clinical symptoms ${ }^{1}$. Recent studies suggest that, metabolism is important for maintaining cartilage function, and aberrant metabolic activities in chondrocytes have been shown in various OA animal models and patients ${ }^{2}$. Despite the severity of $\mathrm{OA}$, the initial triggers of this disease are mostly unknown. Small molecules generated during physiological catabolic reactions often function as damage-associated molecular patterns (DAMP) to activate innate immunity through receptors such as Toll-like receptors (TLR), resulting in enhanced expression of matrix metalloproteinases (MMP) and catabolic factors ${ }^{3}$. Here we studied the effect of various TLR signaling on chondrocyte protein production and mitochondrial oxidative phosphorylation (OXPHOS) We furthermore establish connections between catabolic factors and altered OXPHOS of chondrocytes, aiming to understand the underlying molecular mechanisms.

Objectives: This project is designed to investigate the roles of TLR signaling in the development of $\mathrm{OA}$ and the involved molecular mechanisms Methods: Chondrocytes were obtained from the femural condyles of $\mathrm{OA}$ patients that underwent total knee arthroplasty in the Charite hospital. 3D spheroids were generated and cultured under physioxia $(4.2 \%$ O2) with addition of TGF- $\beta 3$. PGN, Polyl:C, LPS, Flagellin, FSL-I, Imiquimod, ssRNA, or CpG were used as agonist of TLR-1/2, -3, -4, -5, -2/6, -7, -8, or -9 , respectively. Supernatants were collected, and culture medium were renewed twice per week. Pellets were weighted and processed for Alcian blue staining, or mRNA detection, or OXPHOS assay using Seahorse XFe96 Spheroid Mito Stress Test Kit. We used Griess reaction to quantify nitric oxide (NO) in the supernatant and L-NAME to block NO production. We used One-way ANOVA and t test for statistical analysis.

Results: TLR-1/2 and TLR-2/6 stimulation drastically impaired the cartilage matrix production, as they imposed the slowest pellet growth, lowest expression of matrix proteins COL2 and AGC1, but highest expression of matrix degrading enzymes MMP3, ADAMTS5, and the catabolic factor NO. Remarkably, this phenomenon is associated with drastically diminished OXPHOS activity, as shown by the reduction of both basal and maximal respiratory capacity. Moreover, blockade of $\mathrm{NO}$ production reversed the adverse effect imposed by TLR-1/2 stimulation, namely restored their OXPHOS activities and Col2 and AGC1 production in a dose-dependent manner.

Conclusion: Among the whole spectrum of TLR signaling, stimulation of TLR-1/2 and $-2 / 6$ impose the strongest inhibition on chondrocyte pellet growth by suppressing matrix protein synthesis and facilitating matrix degradation. TLR-1/2 stimulation causes the reduction of OXPHOS capacity, which is mediated by the induction of NO. Thus, TLR-1/2 stimulation, via inducing NO production, impairs the mitochondrial respiration in chondrocytes and thus possibly promotes the development of OA.

\section{REFERENCES:}

[1] Piotr Wojdasiewicz. et al. The Role of Inflammatory and Anti-Inflammatory Cytokines in the Pathogenesis of Osteoarthritis. Mediators Inflamm. 2014 561459.

[2] Mobasheri A. et al. The role of metabolism in the pathogenesis of osteoarthritis. Nat Rev Rheumatol. 2017 13(5):302

[3] Orlowsky EW, Kraus VB. The role of innate immunity in osteoarthritis: when our first line of defense goes on the offensive. J Rheumatol. 201542 (3):363

Disclosure of Interests: Ping Shen: None declared, Michael Fuchs: None declared, Marie Reisener: None declared, Clemens Gwinner: None declared, Peihua Wu: None declared, Tobias Jung: None declared, Matthias Pumberger: None declared, Carsten Perka Consultant for: DePuy/ Synthes; Link; Smith\&Nephew; Zimmer, Max Löhning: None declared DOI: 10.1136/annrheumdis-2019-eular.3661 